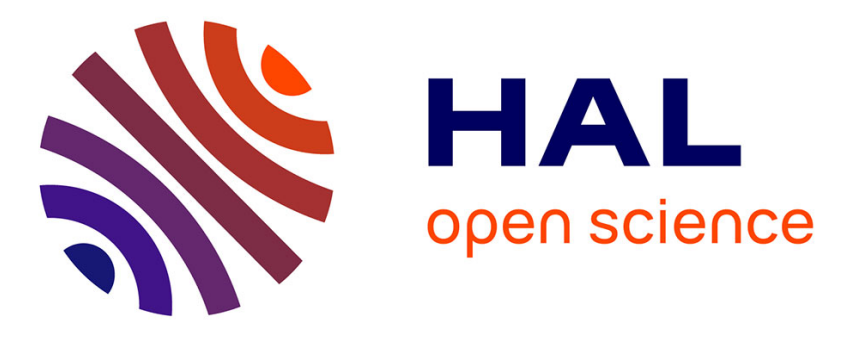

\title{
Peregrine soliton in optical fiber-based systems
}

Bertrand Kibler, Kamal Hammani, Julien Fatome, Christophe Finot, Guy

Millot, Frédéric Dias, Goëry Genty, Nail Akhmediev, John M. Dudley

\section{To cite this version:}

Bertrand Kibler, Kamal Hammani, Julien Fatome, Christophe Finot, Guy Millot, et al.. Peregrine soliton in optical fiber-based systems. Conference on Laser and ElectroOptic, May 2011, Baltimore, United States. pp.QFF1. hal-00568926

\section{HAL Id: hal-00568926 \\ https://hal.science/hal-00568926}

Submitted on 9 May 2011

HAL is a multi-disciplinary open access archive for the deposit and dissemination of scientific research documents, whether they are published or not. The documents may come from teaching and research institutions in France or abroad, or from public or private research centers.
L'archive ouverte pluridisciplinaire HAL, est destinée au dépôt et à la diffusion de documents scientifiques de niveau recherche, publiés ou non, émanant des établissements d'enseignement et de recherche français ou étrangers, des laboratoires publics ou privés. 


\title{
Peregrine soliton in optical fiber-based systems
}

\author{
Bertrand Kibler $^{1}$, Kamal Hammani ${ }^{1}$, Julien Fatome ${ }^{1}$, Christophe Finot ${ }^{1}$, Guy Millot ${ }^{1}$, Frederic Dias ${ }^{2}$, \\ Goery Genty ${ }^{3}$, Neil Akhmediev ${ }^{4}$ and John M. Dudley ${ }^{5}$ \\ ${ }^{1}$ Laboratoire Interdisciplinaire Carnot de Bourgogne, UMR 5209 CNRS-Université de Bourgogne, 9 avenue Alain Savary 21078 Dijon, France \\ ${ }^{2}$ CMLA, ENS Cachan, France; UCD School of Mathematical Sciences, University College Dublin, Belfield, Dublin 4, Ireland \\ ${ }^{3}$ Tampere University of Technology, Optics Laboratory, Tampere, Finland \\ ${ }^{4}$ Optical Sciences Group, Research School of Physics and Engineering, The Australian National University, Canberra ACT 0200, Australia \\ ${ }^{5}$ Institut FEMTO-ST, Université de Franche-Comté, Besançon, France \\ Bertrand.Kibler@u-bourgogne.fr
}

\begin{abstract}
We report the first observation in optics of the Peregrine soliton, a novel class of nonlinear localized structure. Two experimental configurations are explored and the impact of non-ideal initial conditions is discussed.

C2010 Optical Society of America

OCIS codes: (190.0190) Nonlinear optics; (190.5530) Pulse propagation and temporal solitons.
\end{abstract}

\section{Introduction}

Although solitons are central objects of nonlinear science and one of the best known solutions of the nonlinear Schrodinger equation (NLSE), other classes of localized structure are admitted by NLSE including soliton solutions existing upon a finite background [1]. One particular structure of this type is the Peregrine soliton (PS) whose existence was predicted over 25 years ago but surprisingly, it has never been the subject of any systematic experimental study and has never been observed in the original hydrodynamic environment in which it was first studied [2]. The Peregrine soliton is of fundamental significance because it exhibits a two dimensional localization, both in time and space, and because it defines the limit of a wide class of solutions to the NLSE.

In the present contribution, we detail two sets of experiments carried out in optical fibers, which allow us to highlight for the first time the experimental observation of the Peregrine soliton dynamics. Using a wide range of initial conditions, we also show that the process of PS formation is quite robust and on the other hand that a nonideal excitation of the PS structure leads to subpulses splitting beyond the maximum point of compression.

\section{Model}

Light evolution in an optical fiber is appropriately described by the standard NLSE: that can be written in the following normalized form :

$$
i \frac{\partial \psi}{\partial \xi}+\frac{1}{2} \frac{\partial^{2} \psi}{\partial \tau^{2}}+|\psi|^{2} \psi=0
$$

with $\psi$ the complex normalized electric field and $\xi$ and $\tau$ normalized distance and time respectively. We consider here a weakly modulated continuous wave $(\mathrm{CW})$ propagating in an anomalous dispersive fiber. Therefore, the longitudinal evolution is strongly affected by induced modulation instability and the initial sinusoidal field experiences a marked temporal compression. At the low-frequency limit of this process [2,3], a Peregrine soliton is excited, associated with a simple polynomial form describing its two dimensional localization:

$$
\psi(\xi, \tau)=\left[1-\frac{4(1+2 i \xi)}{1+4 \tau^{2}+4 \xi^{2}}\right] e^{i \xi}
$$

\section{Generation in a highly nonlinear fiber at a repetition rate of a several hundreds of GHz}

The experimental set-up we implement is remarkably simple [4] : two external cavity lasers around $1550 \mathrm{~nm}$ create a weakly modulated $\mathrm{CW}$ signal (5\% intensity modulation for a frequency spacing of the laser diodes of $200 \mathrm{GHz}$ ) that is then injected into $900 \mathrm{~m}$ of highly nonlinear fiber $\left(\beta_{2}=-0.885 \mathrm{ps}^{2} \cdot \mathrm{km}^{-1}\right.$ and $\left.\gamma=10 \mathrm{~W}^{-1} \mathrm{~km}^{-1}\right)$. An accurate choice of propagation distance and initial power enables us to approach very close to the ideal compressed shape of the Peregrine soliton. Using frequency-resolved optical gating (FROG), we can compare our experimental measurements with the reshaping of the input field towards the solution predicted by Peregrine [2]. These results 
are shown in Fig. 1a where we plot the temporal intensity and phase profiles of the reshaped field (blue circles) and compare the measurements with the analytic prediction of Peregrine (gray) as well as with realistic simulations using direct integration of the NLSE (red). There is an excellent agreement between experiment, simulation and theory over the central part of the pulse and also in predicting the $\pi$-phase jump observed in the wings (see inset).
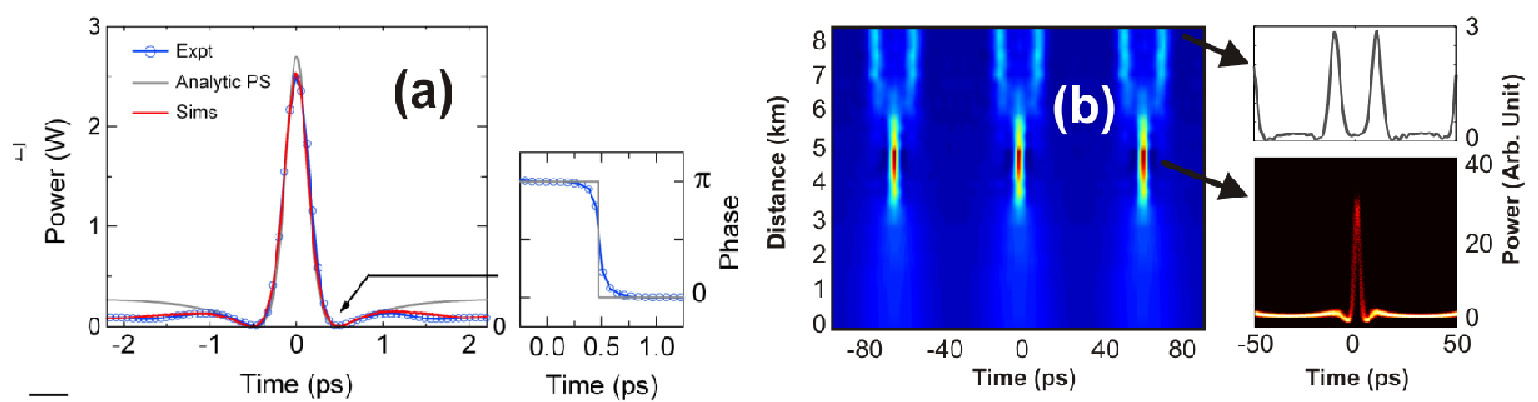

Fig. 1: (a) PS temporal characteristics from experiment (FROG measurements), analysis and simulation. Inset shows a $\pi$-phase jump in vicinity of wings. (b) Longitudinal evolution of temporal profile recorded on an optical sampling oscilloscope (initial power of $0.80 \mathrm{~W}$ ). Insets show PS temporal characteristics from real time observation and the two subpulses at the output of the fiber.

\section{Observation in standard single mode fiber}

To complement the previous observations, we have also tested a second approach where the initial weak modulation is now obtained directly by using a $1550 \mathrm{~nm}$ laser diode that is intensity modulated by a sinusoidal electrical clock at $16 \mathrm{GHz}$. The nonlinear dynamics experienced by the signal is monitored thanks to an ultrafast optical sampling oscilloscope (OSO). The fiber involved in the reshaping process is here a standard SMF-28 with $\beta_{2}=-21.4 \mathrm{ps}^{2} / \mathrm{km}$ and $\gamma=1.2 \mathrm{~W}^{-1} \cdot \mathrm{km}^{-1}$. We have first studied the evolution dynamics over a wide range of initial modulation depth and power and shown that PS localization could be achieved through a large number of initial conditions. Moreover, by combining the real time characterization with cut-back measurements along the whole $8.35 \mathrm{~km}$ of fiber, we provide the first direct observation of the longitudinal dynamics (as shown in Fig. 1b) of this class of soliton. Quite interestingly, we can make out that after the optimum compression point, the PS-like structure experiences a breakup into two subpulses, each possessing similar characteristics of localization upon finite background.

\section{Conclusion}

We have provided here the first measurements of a nonlinear Peregrine soliton structure in any non-discrete NLSE soliton-supporting system. They highlight the existence of a strongly localized temporal peak upon a non-zero background, and confirm Peregrine's theoretical predictions expressed more than 25 years ago. Moreover, a longitudinal experimental scan of the dynamics has clearly outlined the compression and the subsequent splitting occurring because of non-ideal conditions. Our results stress how experiments in optics can be used to conveniently test more general theories of nonlinear waves. In a wider context, the fact that an initial Peregrine soliton can break up into two lower amplitude but equally strongly localized soliton pulses may have important implications for further interpretations of hydrodynamic rogue wave observations as well as establishing new links between optical and hydrodynamic extreme events [5].

\section{References}

[1] N.N. Akhmediev, A. Ankiewicz., Solitons : nonlinear pulses and beams, Chapman \& Hall,, London, 1197.

[2] D.H. Peregrine, Water waves, nonlinear Schrödinger equations and their solutions, J. Aust. Math. Soc. Ser. B, 25 (1983) 16-43.

[3] J.M. Dudley, G. Genty, F. Dias, B. Kibler, N. Akhmediev, Modulation instability, Akhmediev Breathers and continuous wave supercontinuum generation, Opt. Express, 17 (2009) 21497-21508.

[4] B. Kibler, J. Fatome, C. Finot, G. Millot, F. Dias, G. Genty, N. Akhmediev, J.M. Dudley, The Peregrine soliton in nonlinear fibre optics, Nature Physics, 6 (2010) 790-795.

[5] D.R. Solli, C. Ropers, P. Koonath, B. Jalali, Optical rogue waves, Nature, 450 (2007) 1054-U1057. 